\title{
What's your diagnosis
}

respiratory signs is known. Examples include thoracocentesis for pleural space disease, sedation and temperature control with possible intubation or tracheostomy for upper airway disease, bronchodilators and glucocorticoids for feline allergic airway disease, and parenteral frusemide for suspected cardiogenic oedema. If it is not possible to determine the location of the problem, it is recommended to treat for the treatable and to institute a single dose of frusemide, dexamethasone and terbutaline with trial thoracocentesis. Any concurrent lookalikes should also be addressed to improve the patient respiratory pattern.

Look-alike causes of tachypnoea are non-respiratory mechanisms. Examples include decreased oxygen delivery (shock, anaemia, hypoxaemia), metabolic causes (compensation for a metabolic acidosis, hyperthyroidism), neurological disease, drug effect (opioids) and environmental factors (fever, stress, anxiety, pain). Look-alikes are a diagnosis of exclusion and require full evaluation of the patient's history and clinical signs, and the absence of a respiratory cause of signs. If objective measures of oxygenation are available, look-alikes will have normal blood oxygen and levels since the problem is not respiratory in nature.

\section{KEY LEARNING OBJECTIVES}

- Be able to recognize respiratory distress and localize the origin to a part of the respiratory system
- Know which non-invasive patient assessment tools to use to differentiate between the common causes of respiratory distress in cats

- Be able to list non-respiratory look-alike causes of tachypnoea in cats

\section{MULTIPLE CHOICE QUESTIONS}

1. What is the most common cause of respiratory distress in cats?

(A) Congestive heart failure

(B) Pyothorax

(C) Feline allergic airway disease

(D) Neoplasia

2. Which of the following thoracic focused assessment with sonography for trauma (TFAST) findings is consistent with the presence of a pneumothorax in a cat recently hit by a car?
(A) Confluent B-lines
(C) Gator sign
(B) Absent glide sign
(D) Presence of A-lines

3. Which of the following is not a cause of look-alike tachypnoea in cats?
(A) Paracetamol intoxication
(B) Haemoabdomen
(C) Phenobarbital overdose
(D) Diabetic ketoacidosis

\section{Feline abdominal}

masses

\section{Sarah L. Mason}

Abdominal masses are a relatively common presentation in feline patients, often presenting with clinical signs which may include weight loss, inappetence, vomiting and diarrhoea.

The first step in diagnosis is to consider the likely differentials and ideally obtain baseline haematology and biochemistry to assess for red or white blood cell changes (common examples include: anaemia or lymphocytosis in cases of lymphoid neoplasia, hypoalbuminemia and anaemia if gastrointestinal haemorrhage, hyperglobulinaemia in feline infectious peritonitis (FIP)).

Initial investigation of the mass should include abdominal ultrasonography to confirm organ of origin, and fine-needle aspiration of the mass. Slides should be assessed for cellularity in-house to allow for maximum diagnostic capability and avoid a second procedure, however cytology is not always diagnostic. An alternative approach is to take a trucut biopsy. Ultrasonography will often give a good indication of the likelihood of successful resection and guide the decision to proceed with additional staging or surgery. For example, splenic masses are easily treated and diagnosed via splenectomy and histopathology, as opposed to multiple lymph node

masses where biopsy is more likely to be appropriate to differentiate between malignant neoplasia and other abdominal masses (e.g. granuloma).

The most common abdominal mass in cats is intestinal lymphoma. Staging should include testing for feline immunodeficiency virus and feline leukaemia virus, and thoracic radiographs if the budget permits. As lymphoma is a systemic disease, chemotherapy is indicated, regardless of staging and surgical intervention, and so arguably minimal staging is acceptable in financially challenged cases if it will not change a medical treatment plan. In some patients it is of benefit to excise the mass prior to chemotherapy, in the case of obstruction for example.

Other intestinal tumours in felines include carcinoma, mast cell tumour and sarcoma, and these can affect small and large intestine. Splenic and hepatic masses are uncommon in cats and can include mast cell tumour, lymphoid neoplasia and other malignant epithelial and mesenchymal tumours. Abdominal masses may also arise from mesenteric lymph nodes and other organs such as pancreas, bladder or adrenal gland.

Treatment and prognosis are highly variable depending on the aetiology and dissemination of the abdominal mass. Surgical excision is the most appropriate initial therapy for many localized masses and, while the role of chemotherapy is not well documented in non-lymphoid neoplasia, this potentially has benefit in both the adjunctive and gross disease setting. Surgical and medical treatment options will be illustrated using cases within this interactive lecture. 


\section{KEY LEARNING OBJECTIVES}

- Appreciate the differential diagnosis for feline abdominal masses

- Develop the approach to investigation and staging of abdominal masses in cats

- Understand diagnostic and treatment options for gastrointestinal masses including limitations

\section{MULTIPLE CHOICE QUESTIONS}

1. What is the most common presentation of feline intestinal mass?
(A) Lymphoma
(B) Mast cell tumour
(C) Carcinoma
(D) Sarcoma

2. A 2-year-old Siamese cat has a palpable intra-abdominal mass not associated with the intestine, hyperalbuminaemia and peritoneal effusion. What is the most likely diagnosis?
(A) Fungal granuloma
(B) Mast cell tumour
(C) FIP
(D) Adenocarcinoma

3. What is the likely prognosis for feline intestinal mast cell tumour treated with surgery and chemotherapy?
(A) Excellent - around 2 years
(B) Good - around 1 year
(C) Poor - euthanasia is indicated
(D) Variable - depending on presentation and individual

